\title{
Homology of spaces of directed paths on Euclidean cubical complexes
}

\author{
Martin Raussen · Krzysztof Ziemiański
}

Received: 28 February 2013 / Accepted: 6 June 2013 / Published online: 24 July 2013

(C) Tbilisi Centre for Mathematical Sciences 2013

\begin{abstract}
We compute the homology of the spaces of directed paths on a certain class of cubical subcomplexes of the directed Euclidean space $\mathbb{R}^{n}$ by a recursive process. We apply this result to calculate the homology and cohomology of the space of directed loops on the $(n-1)$-skeleton of the directed torus $\vec{T}^{n}$.
\end{abstract}

Keywords Directed paths $\cdot$ Cubical complex $\cdot$ Path space $\cdot$ Homology $\cdot$ Cohomology

Mathematics Subject Classification (2000) $\quad 55 \mathrm{P} 10 \cdot 55 \mathrm{P} 15 \cdot 55 \mathrm{U} 10 \cdot 68 \mathrm{Q} 85$

\section{Introduction}

One of the most important problems of directed algebraic topology is the determination of the homotopy type of spaces of directed paths $\vec{P}(X)_{x}^{y}$ between two points $x, y$ of a directed space $X$. This problem seems to be difficult in general; however several

Dedicated to Hvedri Inassaridze on his 80th birthday

Communicated by Ronald Brown.

The authors gratefully acknowledge support from the European Science Foundation network "Applied and Computational Algebraic Topology" that allowed them to collaborate on this paper during mutual visits made possible by grants 4671 and 5432 .

M. Raussen $(\bowtie)$

Department of Mathematical Sciences, Aalborg University,

Fredrik Bajersvej 7G, 9220 Aalborg Øst, Denmark

e-mail: raussen@math.aau.dk

K. Ziemiański

Faculty of Mathematics Informatics and Mechanics, Warsaw University,

Banacha 2, 02-097 Warszawa, Poland

e-mail: ziemians@mimuw.edu.pl 
results were obtained recently. The first author gave in a series of papers [12-15] a description of the homotopy type $\vec{P}(X)_{x}^{y}$ in the case where $X$ is a directed cube from which collections of homothetic rectangular areas are removed. An alternative description is given in the paper [18] of the second author. The relevant path spaces are shown to be homotopy equivalent to either a simplicial complex or a cubical complex. Even in greater generality, such path spaces have the homotopy type of a CW-complex.

In this paper, we present explicit calculations of the homology and cohomology of directed path spaces in important particular cases in which path spaces can be described as homotopy colimits over simple combinatorial categories; this makes it possible to apply inductive methods.

\section{1 d-spaces}

For a topological space $X$, let $P(X)=X^{I}$ denote the space of all paths in $X$ endowed with the compact-open topology. A d-space [8,9] is a pair $(X, \vec{P}(X))$, where $X$ is a topological space, and $\vec{P}(X) \subseteq P(X)$ is a family of paths on $X$ that contains all constant paths and that is closed under non-decreasing reparametrizations and concatenations. The family $\vec{P}(X)$ is called a $d$-structure on $X$, and paths which belong to $\vec{P}(X)$ will be called directed paths or d-paths. For $x, y \in X$ define the directed path space from $x$ to $y$ as

$$
\vec{P}(X)_{x}^{y}=\{\alpha \in \vec{P}(X): \alpha(0)=x \wedge \alpha(1)=y\} .
$$

The directed real line $\overrightarrow{\mathbb{R}}$ is the d-space with underlying space $\mathbb{R}$ and $\vec{P}(\overrightarrow{\mathbb{R}})$ the set of all non-decreasing paths. Directed Euclidean space $\overrightarrow{\mathbb{R}}^{n}$ is the product $\overrightarrow{\mathbb{R}} \times \cdots \times \overrightarrow{\mathbb{R}}$ with the product d-structure $\vec{P}\left(\overrightarrow{\mathbb{R}}^{n}\right)=\vec{P}(\overrightarrow{\mathbb{R}}) \times \cdots \times \vec{P}(\overrightarrow{\mathbb{R}})$. Finally, the directed torus $\vec{T}^{n}$ is the quotient $\overrightarrow{\mathbb{R}}^{n} / \mathbb{Z}^{n}$; a path on $\vec{T}^{n}$ is directed iff it lifts to a directed path on $\overrightarrow{\mathbb{R}}^{n}$.

\subsection{Euclidean cubical complexes}

An elementary cube in $\mathbb{R}^{n}$ is a product

$$
\left[k_{1}, k_{1}+e_{1}\right] \times \cdots \times\left[k_{n}, k_{n}+e_{n}\right] \subseteq \mathbb{R}^{n}
$$

where $k_{i} \in \mathbb{Z}$ and $e_{i} \in\{0,1\}$; the dimension of a cube is the sum $\sum_{1}^{n} e_{i}$. A Euclidean cubical complex is defined to be a subset $K \subseteq \mathbb{R}^{n}$ that is a union of elementary cubes. The $d$-skeleton of $K$, denoted by $K_{(d)}$, is the union of all elementary cubes contained in $K$ which have dimensions less than or equal to $d$. Euclidean space can be identified with the geometric realization of a suitable pre-cubical set such that realizations of cubes of this pre-cubical set are elementary cubes in $\mathbb{R}^{n}$, and Euclidean cubical sets are the geometric realizations of pre-cubical subsets of that pre-cubical set. Every cubical complex is provided with the directed structure inherited from $\overrightarrow{\mathbb{R}}^{n}$.

Euclidan cubical complexes are special cases of general cubical complexes which are geometric realizations of general pre-cubical sets; cf [1] and the recent book [2]. 


\subsection{Notation}

Points on $\mathbb{R}^{n}$ will be denoted by bold letters, their coordinates by regular ones with suitable indices; for example $\mathbf{a}=\left(a_{1}, \ldots, a_{n}\right)$. Furthermore, we will write $\mathbf{0}$ for $(0, \ldots, 0)$ and $\mathbf{1}$ for $(1, \ldots, 1)$. Three kinds of comparison operators between points of $\mathbb{R}^{n}$ will be used:

$$
\begin{aligned}
& \mathbf{a} \leqslant \mathbf{b} \Leftrightarrow \forall_{i=1}^{n} a_{i} \leqslant b_{i} \\
& \mathbf{a}<\mathbf{b} \Leftrightarrow \forall_{i=1}^{n} a_{i} \leqslant b_{i} \wedge \mathbf{a} \neq \mathbf{b} \\
& \mathbf{a} \ll \mathbf{b} \Leftrightarrow \forall_{i=1}^{n} a_{i}<b_{i} .
\end{aligned}
$$

In analogy to the one-dimensional case, write $[\mathbf{a}, \mathbf{b}]:=\{\mathbf{t}: \mathbf{a} \leqslant \mathbf{t} \leqslant \mathbf{b}\}$ for $\mathbf{a}, \mathbf{b} \in \mathbb{R}^{n}$. Finally, let $|\mathbf{x}|:=\sum_{i=1}^{n}\left|x_{i}\right|$ for $\mathbf{x} \in \mathbb{R}^{n}$ denote the $l_{1}$-norm; the $l_{1}$-metric $\mu(\mathbf{x}, \mathbf{y})=|\mathbf{x}-\mathbf{y}|$ on $\mathbb{R}^{n}$ is compatible with standard Euclidean topology. Notice that $|\mathbf{x}-\mathbf{y}|=|| \mathbf{x}|-| \mathbf{y}||$ whenever $\mathbf{x} \leqslant \mathbf{y}$.

\subsection{The main theorem}

Let $\mathbf{k} \in \mathbb{Z}^{n}, n \geqslant 3$, and let $K \subseteq[\mathbf{0}, \mathbf{k}] \subseteq \mathbb{R}^{n}$ denote a Euclidean cubical complex that contains the $(n-1)$-skeleton $[\mathbf{0}, \mathbf{k}]_{(n-1)} \subseteq K$. A cube sequence in $K$ of length $r$ is a sequence

$$
\left[\mathbf{a}^{*}\right]:=\left[\mathbf{0} \ll \mathbf{a}^{1} \ll \mathbf{a}^{2} \ll \cdots \ll \mathbf{a}^{r} \leqslant \mathbf{k}\right]
$$

where $\mathbf{a}^{i} \in \mathbb{Z}^{n}$ and such that $\left[\mathbf{a}^{i}-\mathbf{1}, \mathbf{a}^{i}\right] \nsubseteq K$. Let $C S_{r}(K)$ be the set of cube sequences of length $r$ and define the graded abelian group $A_{*}(K)$ by

$$
A_{m}(K)= \begin{cases}\mathbb{Z}\left[C S_{m /(n-2)}(K)\right] & \text { if } n-2 \text { divides } m \\ 0 & \text { otherwise. }\end{cases}
$$

The main theorem of this paper is the following

Theorem 1.1 The graded abelian groups $H_{*}(\vec{P}(K))_{\mathbf{0}}^{\mathbf{k}}$ and $A_{*}(K)$ are isomorphic.

1.5 An application: the space of paths on the $(n-1)$-skeleton of the directed torus $\vec{T}^{n}$ Assume that $n \geqslant 3$ and $d \geqslant 2$. Let $\vec{T}_{(d)}^{n}:=\overrightarrow{\mathbb{R}}_{(d)}^{n} / \mathbb{Z}^{n}$ be the $d$-skeleton of $\vec{T}^{n}$. Every directed path $\alpha \in \vec{P}\left(\vec{T}_{(d)}^{n}\right)_{\mathbf{0}}^{\mathbf{0}}$ represents a class $\mathbf{k} \in \pi_{1}\left(\vec{T}_{(d)}^{n}\right)_{\mathbf{0}} \cong \mathbb{Z}^{n}$. By passing to the universal covering of $T_{(d)}^{n}$, we see that $\alpha$ lifts uniquely to a path $\tilde{\alpha} \in \vec{P}\left(\mathbb{R}_{(d)}^{n}\right)_{\mathbf{0}}^{\mathbf{k}}$. Since $\tilde{\alpha}$ is directed, the class $\mathbf{k}$ is non-negative: $\mathbf{0} \leq \mathbf{k}$. Since also directed homotopies lift uniquely, we obtain a homeomorphism

$$
\vec{P}\left(T_{(d)}^{n}\right)_{\mathbf{0}}^{\mathbf{0}} \cong \coprod_{\mathbf{0} \leqslant \mathbf{k} \in \mathbb{Z}^{n}} \vec{P}\left(\mathbb{R}_{(d)}^{n}\right)_{\mathbf{0}}^{\mathbf{k}}
$$


Fig. 1 The space $\mathbf{R}_{(2)}^{3} \cap[\mathbf{0}, \mathbf{2}]$ is homotopy equivalent to the complement of eight cubes within an outer cube $[\mathbf{0}, \mathbf{2}] \subset \mathbf{R}^{3}$. All figures courtesy to E. Haucourt and A. Lang

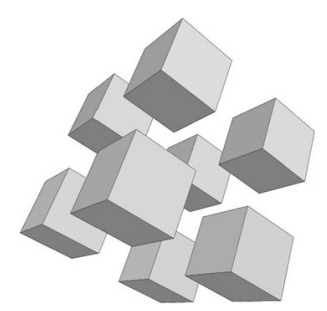

If $d=n-1$, we can apply the main theorem to obtain an isomorphism (Fig. 1)

$$
H_{*}\left(\vec{P}\left(T_{(n-1)}^{n}\right)_{\mathbf{0}}^{\mathbf{0}}\right) \cong \bigoplus_{\mathbf{0} \leqslant \mathbf{k} \in \mathbb{Z}^{n}} A_{*}\left(\mathbb{R}_{(n-1)}^{n} \cap[\mathbf{0}, \mathbf{k}]\right)
$$

The following proposition allows to calculate the Betti numbers of the components:

Proposition 1.2 For $\mathbf{k}=\left(k_{1}, \ldots, k_{n}\right) \geqslant \mathbf{0}$ we get:

$\operatorname{dim} H_{r(n-2)}\left(\vec{P}\left(\mathbb{R}_{(n-1)}^{n}\right)_{\mathbf{0}}^{\mathbf{k}}\right)=\operatorname{dim} A_{r(n-2)}\left(\mathbb{R}_{(n-1)}^{n} \cap[\mathbf{0}, \mathbf{k}]\right)=\left(\begin{array}{c}k_{1} \\ r\end{array}\right)\left(\begin{array}{c}k_{2} \\ r\end{array}\right) \cdots\left(\begin{array}{c}k_{n} \\ r\end{array}\right)$.

Proof The map

$$
\begin{aligned}
& C S_{r}\left(\mathbb{R}_{(n-1)}^{n} \cap[\mathbf{0}, \mathbf{k}]\right) \ni\left[\mathbf{a}^{1} \ll \cdots \ll \mathbf{a}^{r} \leqslant \mathbf{k}\right] \\
& \quad \mapsto\left(\left\{a_{1}^{1}, \ldots, a_{1}^{r}\right\}, \ldots,\left\{a_{n}^{1}, \ldots, a_{n}^{r}\right\}\right) \in 2^{\left\{1, \ldots, k_{1}\right\}} \times \cdots \times 2^{\left\{1, \ldots, k_{n}\right\}}
\end{aligned}
$$

is clearly a bijection. The conclusion follows.

Remark An attempt to calculate the homology of $\vec{P}\left(\mathbb{R}_{2}^{3}\right)_{0}^{(k, l, m)}$ using the poset description for the cell complex of the prod-simplicial complex homotopy equivalent to that path space according to Raussen [13] by "brute force"-even using sophisticated homology software-failed already for $k=l=m=3$. The prod-simplicial complex in this case has dimension $k \operatorname{lm}(n-2)$; its homological dimension is only $\min \{k, l, m\}(n-2)$. This contrast was one of the motivations for looking for better descriptions of path spaces.

\subsection{Euclidean cubical complexes and concurrency}

One of the motivations for developing directed algebraic topology goes back to particular models in concurrency theory, the so-called higher dimensional automata, cf, e.g. $[10,17]$. A particular class of higher dimensional automata arises from semaphore or mutex models: Each processor records on a time line when it accesses $(\mathrm{P})$ and relinquishes (V) a number of shared objects; the forbidden region $F$ associated to such a PV-program (cf [4]) consists of a union of isothetic hyperrectangles $R^{i} \subset \vec{I}^{n}$ within an $n$-cube $\vec{I}^{n} \subset \overrightarrow{\mathbb{R}}^{n} ; \operatorname{cf}[7]$. 

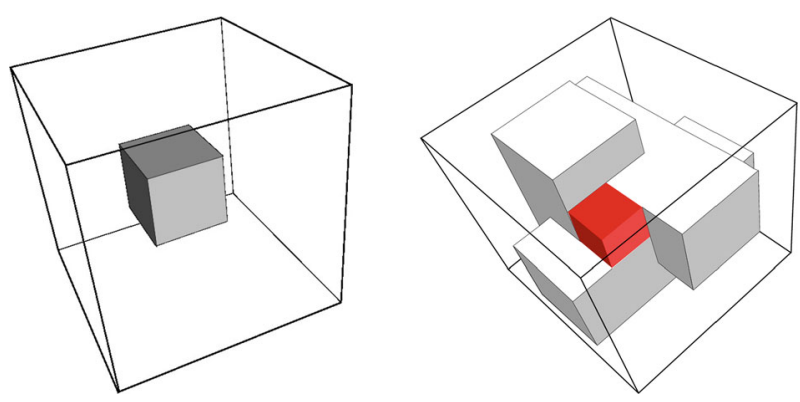

Fig. 2 Left Forbidden region corresponding to one semaphore of arity 2 in the presence of 3 processors. Right Forbidden (and unsafe) region corresponding to 3 dining philosophers, cf [5]

The particular Euclidean complexes whose path spaces we study correspond to PV-programs with the following two particular properties:

- All shared objects have arity $n-1$, ie, $n-1$ out of $n$ but not all $n$ processes can access the object at any given time (Fig. 2);

- The PV-program for every indiviudal processor is of type $(P V)(P V) \cdots(P V)-\mathrm{a}$ variety of shared objects is allowed. In particular, every access to a shared object is terminated before a new one is accessed. This has the consequence that the hyperretangles $R^{i}$ and their projections to the axes do not overlap with each other.

No doubt that this represents a very particular case. On the other hand, our result seems to be the first non-trivial calculation of the homology of spaces of directed paths in closed form. Note that a description of a simplicial complex homotopy equivalent to directed paths in a torus with holes was obtained in Fajstrup [6].

The application in Sect. 1.5-which motivated this line of investigation-shows that it is also possible to consider programs with loops. The case considered here corresponds to $n$ looped processors of type $(P a V a)^{*}\|\cdots\|(P a V a)^{*}$.

\subsection{The case $n=2$}

Only for $n=2$, the path spaces are, in general, not connected - and therefore the result of a distributed programme may depend on the schedule. The method described above still works, but there is a slight twist due to the fact that cube sequences regardless of their length all contribute (only) to dimension 0 :

The space $\vec{P}(K)_{\mathbf{0}}^{\mathbf{k}}$ is a space consisting of contractible components. The number of components is $\beta_{0}\left(\vec{P}(K)_{\mathbf{0}}^{\mathbf{k}}\right)=|C S(K)|+1$, the number of all cube sequences in $K$ augmented by one. The reason is that both sides in the equation above obey to the

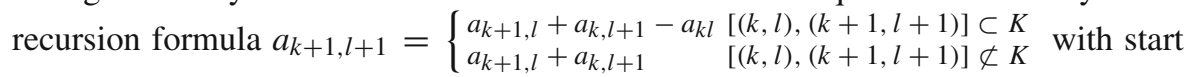
values $a_{k, 0}=a_{0, l}=1$.

In the particular case dealt with in Sect. 1.5, we obtain: $\beta_{0}\left(\vec{P}\left(\mathbb{R}_{1}^{2}\right)_{\mathbf{0}}^{(k, l)}\right)=\left(\begin{array}{c}k+l \\ k\end{array}\right)$. 
1.8 Outline of the paper

In Sect. 2 we construct, for an arbitrary Euclidean cubical complex $K$, a homotopy equivalence between $\vec{P}(K)_{\mathbf{0}}^{\mathbf{k}}$ and a certain homotopy colimit of spaces which are homotopy equivalent to one of the "smaller" spaces $\vec{P}(K)_{\mathbf{0}}^{\mathbf{l}}$ for $\mathbf{l}<\mathbf{k}$. In Sect. 3, we construct a homomorphism $A_{*}(K) \rightarrow H_{*}\left(\vec{P}(K)_{\mathbf{0}}^{\mathbf{k}}\right)$. Then we prove, under the assumption that $[\mathbf{0}, \mathbf{k}]_{(n-1)} \subseteq K$, that this homomorphism is actually an isomorphism. The proof is inductive and uses the homotopy colimit description from Sect. 2. In Sect. 4, we determine the cohomology ring structure $H^{*}\left(\vec{P}(K)_{\mathbf{0}}^{\mathbf{k}}\right)$.

\section{A recursive description of path spaces}

In this section we construct a presentation of directed paths spaces on a Eulidean cubical complex as a homotopy colimit of path spaces of certain subcomplexes. Fix $\mathbf{0} \ll \mathbf{k} \in \mathbb{Z}^{n}$ and a cubical complex $K \subseteq[\mathbf{0}, \mathbf{k}]$.

\subsection{A transversal section}

Fix $\varepsilon \in(0,1)$. Let $S(K)=\{\mathbf{x} \in K:|\mathbf{x}|=|\mathbf{k}|-\varepsilon\} \subset S=\left\{\mathbf{x} \in \mathbb{R}^{n}|| \mathbf{x}|=| \mathbf{k} \mid-\varepsilon\right\}$.

Proposition 2.1 For every path $\alpha \in \vec{P}(K)_{\mathbf{0}}^{\mathbf{k}}$ there exists a unique $s(\alpha) \in S(K)$ belonging to the image of $\alpha$. Moreover, the map $s: \vec{P}(K)_{\mathbf{0}}^{\mathbf{k}} \rightarrow S(K)$ is continuous (with respect to the compact-open topology on $\left.\vec{P}(K)_{\mathbf{0}}^{\mathbf{k}}\right)$.

Proof Since $|\alpha(0)|=0,|\alpha(1)|=|\mathbf{k}|$, there exists $t_{\alpha}$ such that $\alpha\left(t_{\alpha}\right)=|\mathbf{k}|-\varepsilon$. If $\left|\alpha\left(t_{\alpha}\right)\right|=\left|\alpha\left(t_{\alpha}^{\prime}\right)\right|$ for $t_{\alpha}<t_{\alpha}^{\prime}$, then $\alpha\left(t_{\alpha}\right) \leq \alpha\left(t_{\alpha}^{\prime}\right)$. This implies that $\left|\alpha\left(t_{\alpha}\right)-\alpha\left(t_{\alpha}^{\prime}\right)\right|=$ ||$\alpha\left(t_{\alpha}\right)|-| \alpha\left(t_{\alpha}^{\prime}\right)||=0$. Therefore $s(\alpha)$ is uniquely determined. In the parlance of Raussen [11], the subset $S(X)$ is both achronal and unavoidable from $\mathbf{0}$ to $\mathbf{k}$.

To prove the continuity of $s$, it is sufficient to show that the inverse images of open balls $B(\mathbf{x}, r)=\{\mathbf{y} \in K:|\mathbf{x}-\mathbf{y}|<r\}$ are open in $\vec{P}(K)_{\mathbf{0}}^{\mathbf{k}}$ : Fix $\mathbf{x} \in K, r>0$, $\alpha \in s^{-1}(B(\mathbf{x}, r))$ and $t_{\alpha} \in I$ such that $\alpha\left(t_{\alpha}\right)=s(\alpha)$. Let $\beta \in \vec{P}(K)_{\mathbf{0}}^{\mathbf{k}}$ be a path such that $\left|\beta\left(t_{\alpha}\right)-s(\alpha)\right|<r^{\prime}$, where $r^{\prime}=(r-|\mathbf{x}-s(\alpha)|) / 2$. Since $s(\beta)$ and $\beta\left(t_{\alpha}\right)$ are comparable, we have

$$
\left|\beta\left(t_{\alpha}\right)-s(\beta)\right|=|| \beta\left(t_{\alpha}\right)|-| s(\beta)||=|| \beta\left(t_{\alpha}\right)|-| s(\alpha)||<r^{\prime} .
$$

Finally,

$|\mathbf{x}-s(\beta)| \leqslant|\mathbf{x}-s(\alpha)|+\left|s(\alpha)-\beta\left(t_{\alpha}\right)\right|+\left|\beta\left(t_{\alpha}\right)-s(\beta)\right|<|\mathbf{x}-s(\alpha)|+2 r^{\prime}<r$,

i. e. $\beta \in s^{-1}(B(\mathbf{x}, r))$. Hence the set $\left\{\beta \in \vec{P}(K)_{\mathbf{0}}^{\mathbf{k}}: \beta\left(t_{\alpha}\right) \in B\left(s(\alpha), r^{\prime}\right)\right\}$ is an open neighbourhood of $\alpha$ contained in $s^{-1}(B(\mathbf{x}), r)$. 


\subsection{A description of $S(K)$}

The map

$$
R: S \ni \mathbf{t} \mapsto \varepsilon^{-1}(\mathbf{k}-\mathbf{t}) \in\{\mathbf{x}: \mathbf{x} \geqslant 0 \wedge|\mathbf{x}|=1\}=\left|\Delta^{n-1}\right|
$$

is a homeomorphism from $S$ to the standard simplex $\Delta^{n-1}$. It maps $S(K)$ homeomorphically onto a simplicial subcomplex $\Delta_{K} \subset \Delta^{n-1}$.

The category $\Delta_{n-1}^{o p}$ of subsimplices of $\Delta_{n-1}$ can be identified by an isomorphism of categories with the (inverse) poset category $\mathcal{J}_{n-1}$ of sequences $\mathbf{j} \in\{0,1\}^{n}$ with $\mathbf{j} \neq \mathbf{0}$. Such a sequence $\mathbf{j}$ corresponds to the subsimplex

$$
\Delta_{\mathbf{j}}=\left\{\mathbf{t} \in \Delta_{n-1}: \forall_{i=1}^{n} \quad j_{i}=0 \Rightarrow t_{i}=0\right\} \subseteq \Delta_{n-1} .
$$

The morphism $\mathbf{j} \rightarrow \mathbf{j}^{\prime}$ (for every $\mathbf{j} \geqslant \mathbf{j}^{\prime}$ ) corresponds to the inclusion $\Delta_{\mathbf{j}} \subset \Delta_{\mathbf{j}^{\prime}}$.

The restriction of this correspondence to the category of subsimplices of $\Delta_{K}$ provides an isomorphism between that category and the full subcategory $\mathcal{J}_{K} \subset \mathcal{J}_{n-1}$ with objects

$$
\mathrm{Ob}\left(\mathcal{J}_{K}\right):=\left\{\mathbf{0}<\mathbf{j} \in\{0,1\}^{n}: \mathbf{j} \subseteq \Delta_{K}\right\}=\left\{\mathbf{j} \in\{0,1\}^{n}:[\mathbf{k}-\mathbf{j}, \mathbf{k}] \subseteq K\right\}
$$

Two cases will be of particular importance:

$$
\mathcal{J}_{K}= \begin{cases}\mathcal{J}_{n-1} & {[\mathbf{k}-\mathbf{1}, \mathbf{k}] \subset K} \\ \hat{\mathcal{J}}_{n-1}:=\mathcal{J}_{n-1} \backslash\{\mathbf{1}\} & {[\mathbf{k}-\mathbf{1}, \mathbf{k}] \cap K=\partial[\mathbf{k}-\mathbf{1}, \mathbf{k}] .}\end{cases}
$$

\subsection{A cover of $\vec{P}(K)_{\mathbf{0}}^{\mathbf{k}}$}

The geometric realization of $S(K)$ can be covered by stars of its vertices and this cover lifts to a cover of $\vec{P}(K)_{\mathbf{0}}^{\mathbf{k}}$. For every $\mathbf{j} \in \mathcal{J}_{K}$ define

$$
F_{\mathbf{j}} \vec{P}(K)_{\mathbf{0}}^{\mathbf{k}}:=(R \circ s)^{-1}(\operatorname{st}(\mathbf{j}))=\left\{\alpha \in \vec{P}(K)_{\mathbf{0}}^{\mathbf{k}}: \forall_{i=1}^{n} j_{i}=1 \Rightarrow s(\alpha)_{i}<k_{i}\right\}
$$

The spaces $F_{\mathbf{j}} \vec{P}(K)_{\mathbf{0}}^{\mathbf{k}}$ clearly cover all of $\vec{P}(K)_{\mathbf{0}}^{\mathbf{k}}$. The cover $\left\{F_{\mathbf{j}} \vec{P}(K)_{\mathbf{0}}^{\mathbf{k}}\right\}$ is closed under intersections since $F_{\mathbf{j}} \vec{P}(K)_{\mathbf{0}}^{\mathbf{k}} \cap F_{\mathbf{j}^{\prime}} \vec{P}(K)_{\mathbf{0}}^{\mathbf{k}}=F_{\mathbf{j} \cup \mathbf{j}^{\prime}} \vec{P}(K)_{\mathbf{0}}^{\mathbf{k}}$, where $\left(\mathbf{j} \cup \mathbf{j}^{\prime}\right)_{i}=$ $\max \left\{j_{i}, j_{i}^{\prime}\right\}$. Moreover the category associated with this cover is precisely $\mathcal{J}_{K}$. As a consequence, cf [16, Proposition 4.1], the inclusions $F_{\mathbf{j}} \vec{P}(K)_{\mathbf{0}}^{\mathbf{k}} \subseteq \vec{P}(K)_{\mathbf{0}}^{\mathbf{k}}$ induce a homotopy equivalence

$$
\operatorname{hocolim}_{\mathbf{j} \in \mathcal{J}_{K}} F_{\mathbf{j}} \vec{P}(K)_{\mathbf{0}}^{\mathbf{k}} \longrightarrow \vec{P}(K)_{\mathbf{0}}^{\mathbf{k}}=\operatorname{colim}_{\mathbf{j} \in \mathcal{J}_{K}} F_{\mathbf{j}} \vec{P}(K)_{\mathbf{0}}^{\mathbf{k}} .
$$

The next step is to prove that $F_{\mathbf{j}} \vec{P}(K)_{\mathbf{0}}^{\mathbf{k}}$ is homotopy equivalent to $\vec{P}(K)_{\mathbf{0}}^{\mathbf{k}-\mathbf{j}}$. This will enable us to use the decomposition (2.2) for inductive calculations of path spaces. But first, we need some technical lemmas which will be presented in a greater generality. 
2.4 Past deformation retractions

Definition 2.2 Let $X$ be a d-space with a subspace $Y \subseteq X$. A past deformation retraction of $X$ onto $Y$ is a d-map $d: X \times \vec{I} \rightarrow X$ (preserving d-structures; cf [9]) such that

- $r(x):=d(x, 0) \in Y$ for every $x \in X$,

- $d(x, 1)=x$ for every $x \in X$,

- $d(y, t)=y$ for every $y \in Y$ and every $t \in I$.

Proposition 2.3 If $d: X \times \vec{I} \rightarrow X$ is a past deformation retraction on $Y$, then for every $x \in X$ and $y \in Y$ the maps

$$
\begin{gathered}
F: \vec{P}(Y)_{y}^{d(x, 0)} \ni \alpha \mapsto \alpha * d(x,-) \in \vec{P}(X)_{y}^{x} \\
G: \vec{P}(X)_{y}^{x} \ni \alpha \mapsto d(\alpha, 0) \in \vec{P}(Y)_{y}^{d(x, 0)}
\end{gathered}
$$

are mutually inverse homotopy equivalences.

Proof A homotopy $H$ between $I d_{\vec{P}(Y)_{y}^{d(x, 0)}}$ and $G \circ F$ is given by the formula

$$
H(\alpha, s)(t)= \begin{cases}\alpha\left(t(1-s / 2)^{-1}\right) & \text { for } 0 \leqslant t \leqslant 1-s / 2 \\ d(x, 0) & \text { for } 1-s / 2 \leqslant t \leqslant 1\end{cases}
$$

and a homotopy $H^{\prime}$ between $I d_{\vec{P}(X)_{y}^{x}}$ and $F \circ G$ by

$$
H^{\prime}(\beta, s)(t)= \begin{cases}d\left(\beta\left(t(1-s / 2)^{-1}\right), 1-s\right) & \text { for } 0 \leqslant t \leqslant 1-s / 2 \\ d(x, 2 t-1) & \text { for } 1-s / 2 \leqslant t \leqslant 1\end{cases}
$$

$2.5 F_{\mathbf{j}} \vec{P}(K)_{\mathbf{0}}^{\mathbf{k}}$ up to homotopy

For $\mathbf{j} \in \mathcal{J}_{K}$ define

$$
\begin{aligned}
& X_{\mathbf{j}}:=\left\{\mathbf{t} \in K: \forall_{i: \mathbf{j}_{i}=1} t_{i}<k_{i}\right\} \\
& K_{\mathbf{j}}:=\{\mathbf{t} \in K: \mathbf{t} \leqslant \mathbf{k}-\mathbf{j}\}=K \cap[\mathbf{0}, \mathbf{k}-\mathbf{j}]
\end{aligned}
$$

and let $\bar{X}_{\mathbf{j}}$ be the closure of $X_{\mathbf{j}}$ in $K$.

Proposition $2.4 K_{\mathbf{j}}$ is a past deformation retract of $\bar{X}_{\mathbf{j}}$.

Proof Every $\mathbf{t} \in \bar{X}_{\mathbf{j}}$ belongs to some cube $[\mathbf{c}, \mathbf{d}] \subseteq K, \mathbf{c}, \mathbf{d} \in \mathbb{Z}^{n}$ whose interior is contained in $X_{\mathbf{j}}$. It implies that $c_{i}=k_{i}-1$ for every $i$ such that $d_{i}=k_{i}$ and $j_{i}=1$. Define the retraction $r^{\mathbf{j}}: \bar{X}_{\mathbf{j}} \rightarrow K_{\mathbf{j}}$ by the formula

$$
r_{i}^{\mathbf{j}}(\mathbf{t})= \begin{cases}t_{i} & \text { for } t_{i} \leqslant k_{i}-j_{i} \\ k_{i}-j_{i} & \text { for } t_{i} \geqslant k_{i}-j_{i}\end{cases}
$$


and the deformation between $r^{\mathbf{j}}$ and identity by convex combination. Since both $\mathbf{t}$ and $r^{\mathbf{j}}(\mathbf{j})$ belong to the cube $[\mathbf{c}, \mathbf{d}]$ the map $r^{\mathbf{j}}$ is well-defined.

Proposition 2.5 The image $\alpha(I)$ associated to any path $\alpha \in F_{\mathbf{j}} \vec{P}(K)_{\mathbf{0}}^{\mathbf{k}}$ is contained in $\bar{K}_{\mathbf{j}}$.

Proof Let $\alpha \in F_{\mathbf{j}} \vec{P}(K)_{\mathbf{0}}^{\mathbf{k}}$ and let $t_{\alpha} \in I$ satisfies $\alpha\left(t_{\alpha}\right)=s(\alpha)$. For $t \leqslant t_{\alpha}$, we have $\alpha(t)_{i} \leqslant \alpha\left(t_{\alpha}\right)_{i}<k_{i}$ whenever $\mathbf{j}_{i}=1$. Then $\alpha\left(\left[0, t_{\alpha}\right]\right) \subseteq K_{\mathbf{j}} \subseteq \bar{K}_{\mathbf{j}}$. If $t>t_{\alpha}$, then $\alpha(t) \in\left[\mathbf{k}-\mathbf{j}^{\prime}, \mathbf{k}\right]$, where $\left[\mathbf{k}-\mathbf{j}^{\prime}, \mathbf{k}\right]$ is a minimal cube containing $s(\alpha)$ in its interior. As a consequence, $\alpha\left(\left[t_{\alpha}, 1\right]\right) \subseteq \bar{K}_{\mathbf{j}}$ and hence $F_{\mathbf{j}} \vec{P}(K)_{\mathbf{0}}^{\mathbf{k}} \subseteq \vec{P}\left(\bar{K}_{\mathbf{j}}\right)_{\mathbf{0}}^{\mathbf{k}}$.

For every cube $[\mathbf{c}, \mathbf{d}] \subseteq K$ let $i_{\mathbf{c}}^{\mathbf{d}}: \vec{P}(K)_{\mathbf{0}}^{\mathbf{c}} \rightarrow \vec{P}(K)_{\mathbf{0}}^{\mathbf{d}}$ denote the concatenation with the linear path $t \mapsto(1-t) \mathbf{c}+t \mathbf{d}$. Note that $i_{\mathbf{k}-\mathbf{j}}^{\mathbf{k}}\left(\vec{P}(K)_{\mathbf{0}}^{\mathbf{k}-\mathbf{j}}\right) \subseteq F_{\mathbf{j}} \vec{P}(K)_{\mathbf{0}}^{\mathbf{k}}$ for $\mathbf{j} \in \mathcal{J}_{K}$.

Proposition 2.6 For every $\mathbf{j} \in \mathcal{J}_{K}$ the map $i_{\mathbf{k}-\mathbf{j}}^{\mathbf{k}}: \vec{P}\left(K_{\mathbf{j}}\right)_{\mathbf{0}}^{\mathbf{k}-\mathbf{j}} \rightarrow F_{\mathbf{j}} \vec{P}(K)_{\mathbf{0}}^{\mathbf{k}}$ is a homotopy equivalence. Moreover, for every morphism $\mathbf{j}^{\prime} \rightarrow \mathbf{j}$ in $\mathcal{J}_{K}$ the diagram

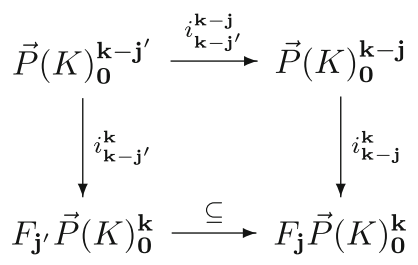

commutes up to homotopy.

Proof The map $i_{\mathbf{k}-\mathbf{j}}^{\mathbf{k}}$ is a homotopy equivalence by Proposition 2.3. The commutativity of the diagram is obvious from the definitions.

\subsection{Specific path spaces}

\subsubsection{Boundary of a cube}

Let $\mathbf{k}=\mathbf{1}$ and let $K=[\mathbf{0}, \mathbf{1}]_{(n-1)}$. Then $S(K) \simeq \partial \Delta^{n-1}$ and $\mathcal{J}_{K}=\mathcal{J}_{n-1} \backslash\{\mathbf{1}\}$ with objects $\left\{\mathbf{j} \in\{0,1\}^{n}: 0<\sum_{i=1}^{n} j_{i}<n\right\}$. Furthermore, for every such $\mathbf{j}$, $F_{\mathbf{j}} \vec{P}(K)_{\mathbf{0}}^{\mathbf{1}} \simeq \vec{P}(K)_{\mathbf{0}}^{\mathbf{k}-\mathbf{j}}=\vec{P}[\mathbf{0}, \mathbf{k}-\mathbf{j}]_{\mathbf{0}}^{\mathbf{k}-\mathbf{j}}$ is contractible since it contains $\{\mathbf{0}\}$ as a past deformation retract, cf Proposition 2.3. As a consequence,

$$
\vec{P}\left(\mathbb{R}_{(n-1)}^{n}\right)_{\mathbf{0}}^{\mathbf{1}} \cong \operatorname{hocolim}_{\mathbf{j} \in \mathcal{J}(K)} F_{\mathbf{j}} \vec{P}(K)_{\mathbf{0}}^{\mathbf{k}-\mathbf{j}} \simeq N \mathcal{J}(K) \cong \partial \Delta^{n-1} \simeq S^{n-2}
$$

Remark This result is also an immediate consequence of Raussen [13, Corollary 4.12].

For the remaining part of the paper, we fix a generator $x_{\mathbf{1}} \in H_{n-2}\left(\vec{P}\left([\mathbf{0}, \mathbf{1}]_{(n-1)}\right)_{\mathbf{0}}^{\mathbf{1}}\right) \cong$ $H_{n-2}(|N \mathcal{J}|)$. By shifting, we obtain the generators $x_{\mathbf{k}} \in H_{n-2}\left(\vec{P}\left([\mathbf{k}-\mathbf{1}, \mathbf{k}]_{(n-1)}\right)_{\mathbf{k}-\mathbf{1}}^{\mathbf{k}}\right)$ for $\mathbf{k} \in \mathbb{Z}^{n}$. 


\subsubsection{Connectivity of certain path spaces}

Proposition 2.7 If a subcomplex $K \subseteq[\mathbf{0}, \mathbf{k}]$ contains the 2-skeleton of $[0, \mathbf{k}]$, then $\vec{P}(K)_{\mathbf{0}}^{\mathbf{k}}$ is connected.

Proof This is obvious if either $\mathbf{k}=\mathbf{0}$, or $n=2$. Assume that the conclusion holds for all complexes $K^{\prime} \subseteq\left[\mathbf{0}, \mathbf{k}^{\prime}\right], \mathbf{k}^{\prime} \in \mathbb{Z}^{n^{\prime}}$, such that $n^{\prime} \leqslant n$ or $n^{\prime}=n$ and $\mathbf{k}^{\prime}<\mathbf{k}$.

For $\mathbf{k}>\mathbf{0}, S(K) \subseteq \Delta^{n-1}$ contains the 1-skeleton of $\Delta^{n-1}$ and is therefore connected. Then

$$
\vec{P}(K)_{\mathbf{0}}^{\mathbf{k}} \simeq \operatorname{hocolim}_{\mathbf{j} \in \mathcal{J}_{K}} F_{\mathbf{j}} \vec{P}(K)_{\mathbf{0}}^{\mathbf{k}}
$$

is connected, because it is a homotopy colimit of connected spaces $F_{\mathbf{j}} \vec{P}(K)_{\mathbf{0}}^{\mathbf{k}} \simeq$ $\vec{P}(K)_{\mathbf{0}}^{\mathbf{k}-\mathbf{j}}=\vec{P}(K \cap[\mathbf{0}, \mathbf{k}-\mathbf{j}])_{\mathbf{0}}^{\mathbf{k}-\mathbf{j}}$ (by the inductive assumption) over a connected category $\mathcal{J}_{K}$.

\section{Homology of the path space $\vec{P}(K)_{0}^{\mathrm{k}}$}

Fix $n \geqslant 3, \mathbf{k} \in \mathbb{Z}^{n}$. Let $K \subseteq[\mathbf{0}, \mathbf{k}] \subseteq \mathbb{R}^{n}$ denote a Euclidean cubical complex which contains the $(n-1)$-skeleton of $[\mathbf{0}, \mathbf{k}]$. We will define a homomorphism $\Phi_{K}$ : $A_{*}(K) \rightarrow H_{*}\left(\vec{P}(K)_{\mathbf{0}}^{\mathbf{k}}\right)$ from the graded abelian group $A_{*}(K)$ defined in Sect. 1.4 into the homology of the path space and prove that it is an isomorphism.

\subsection{The homomorphism $\Phi_{K}$}

\subsubsection{Definitions}

For every cube sequence $\mathbf{a}^{*}=\left[\mathbf{0} \ll \mathbf{a}^{1} \ll \cdots \ll \mathbf{a}^{r}\right] \in C S_{r}(K)$ in $K$ choose paths $\beta_{i} \in \vec{P}(K)_{\mathbf{a}^{i}}^{\mathbf{a}^{i+1}-\mathbf{1}}, i=0, \ldots, r$ (we assume $\mathbf{a}^{0}=\mathbf{0}, \mathbf{a}^{r+1}=\mathbf{k}+\mathbf{1}$ ). Let $c\left(\mathbf{a}^{*}\right)$ be the following concatenation map

$$
c\left(\mathbf{a}^{*}\right): \prod_{j=1}^{r} \vec{P}(K)_{\mathbf{a}^{j-1}}^{\mathbf{a}^{j}} \ni\left(\alpha_{j}\right) \mapsto \beta_{0} * \alpha_{1} * \beta_{1} * \cdots * \alpha_{r} * \beta_{r} \in \vec{P}(K)_{\mathbf{0}}^{\mathbf{k}} .
$$

Then define $\Phi_{K}$ on generators by

$$
\Phi_{K}\left(\left[\mathbf{a}^{1} \ll \cdots \ll \mathbf{a}^{r}\right]\right):=c\left(\mathbf{a}^{*}\right)_{*}\left(x_{\mathbf{a}^{1}} \times x_{\mathbf{a}^{2}} \times \cdots \times x_{\mathbf{a}^{r}}\right) \in H_{*}\left(\vec{P}(K)_{\mathbf{0}}^{\mathbf{k}}\right),
$$

and extend as a homomorphism.

The element $\mathbf{x}_{\mathbf{a}^{i}} \in H_{n-2}\left(\vec{P}(K)_{\mathbf{a}^{j}-\mathbf{1}}^{\mathbf{a}^{j}}\right) \cong H_{n-2}\left(\vec{P}\left(\partial\left[\mathbf{a}^{j}-\mathbf{1}, \mathbf{a}^{j}\right]\right)_{\mathbf{a}^{j}-\mathbf{1}}^{\mathbf{a}^{j}}\right)$ is a generator chosen as in Sect. 2.6.1. According to Proposition 2.7, the map $c\left(\mathbf{a}^{*}\right)$ does not depend (up to homotopy) on the choice of the paths $\beta_{i}$, and this implies that $\Phi_{K}$ is well-defined. 


\subsubsection{Naturality}

Let $\mathbf{l} \leqslant \mathbf{k}$ and let $L \subseteq[\mathbf{0}, \mathbf{l}]$ be a cubical complex such that $[\mathbf{0}, \mathbf{l}]_{(n-1)} \subseteq L \subseteq K \cap[\mathbf{0}, \mathbf{l}]$. With respect to the homomorphism given by

$$
\varphi_{L}^{K}: A_{*}(L) \ni\left[\mathbf{a}^{*}\right] \mapsto\left\{\begin{array}{ll}
{\left[\mathbf{a}^{*}\right]} & \text { if }\left[\mathbf{a}^{*}\right] \text { is a cube sequence in } K \\
0 & \text { otherwise. }
\end{array} \in A_{*}(K),\right.
$$

the homomorphism $\Phi_{K}$ is natural in the following sense:

Proposition 3.1 The diagram

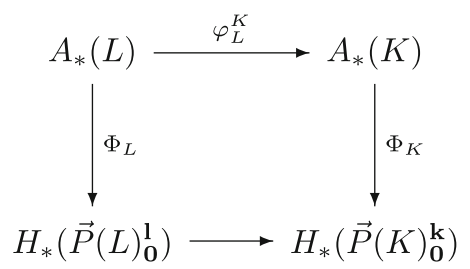

is commutative. The bottom map is induced by the concatenation with a fixed directed path $\alpha \in \vec{P}(K)_{\mathbf{l}}^{\mathbf{k}}$.

Proof Straightforward from the definitions.

\subsection{The main theorem}

The main result of this section is the following

Theorem 3.2 For every Euclidean cubical complex $[\mathbf{0}, \mathbf{k}]_{(n-1)} \subseteq K \subseteq[\mathbf{0}, \mathbf{k}], \mathbf{k} \in$ $\mathbb{Z}^{n}, \mathbf{k} \geq \mathbf{0}, n>2$, the homomorphism $\Phi_{K}: A_{*}(K) \rightarrow H_{*}\left(\vec{P}(K)_{\mathbf{0}}^{\mathbf{k}}\right)$ is an isomorphism of graded abelian groups.

The proof is by induction on $\mathbf{k}$. To start the induction, notice that if $\prod_{i} k_{i}=0$, then both $H_{*}(K)_{\mathbf{0}}^{\mathbf{k}}$ and $A_{*}(K)$ are isomorphic to $(\mathbb{Z}, 0)$, the homology of a point, since $[\mathbf{0}, \mathbf{k}]_{(n-1)} \subseteq K$. Let us assume that Theorem 3.2 is valid for all Euclidean cubical complexes contained in $[\mathbf{0}, \mathbf{l}]$ for $\mathbf{l}<\mathbf{k}$.

Since $K$ is assumed to contain the $(n-1)$-skeleton of $[\mathbf{0}, \mathbf{k}]$, there are only two cases to consider: either $[\mathbf{k}-\mathbf{1}, \mathbf{k}]$ is contained in $K$, or it is not; in that case $[\mathbf{k}-\mathbf{1}, \mathbf{k}] \cap K=$ $\partial[\mathbf{k}-\mathbf{1}, \mathbf{k}]$. For simplicity, we will write $\mathcal{J}=\mathcal{J}_{n-1}$, resp. $\hat{\mathcal{J}}=\hat{\mathcal{J}}_{n-1}$ for the relevant categories; cf Sect. 2.2. Let $\mathbf{A} \mathbf{b}_{*}$ be the category of graded abelian groups.

\subsubsection{The case $[\mathbf{k}-\mathbf{1}, \mathbf{k}] \subseteq K$}

The objects of the category $\mathcal{J}$ are all $n$-tuples $\mathbf{0}<\mathbf{j} \in\{0,1\}^{n}$, cf (2.1). For $\mathbf{j} \in$ $\{0,1\}^{n}$ denote $K_{\mathbf{j}}:=K \cap[\mathbf{0}, \mathbf{k}-\mathbf{j}]$. Notice that for any morphism $\mathbf{j} \rightarrow \mathbf{j}^{\prime}$ in $\mathcal{J}$, the 
homomorphisms

$$
\begin{aligned}
\varphi_{K_{\mathbf{j}}}^{K_{\mathbf{j}^{\prime}}}: & A_{*}\left(K_{\mathbf{j}}\right) \rightarrow A_{*}\left(K_{\mathbf{j}^{\prime}}\right) \\
\left(i_{\mathbf{k}-\mathbf{j}}^{\mathbf{k}-\mathbf{j}^{\prime}}\right)_{*}: & H_{*}\left(\vec{P}\left(K_{\mathbf{j}}\right)_{\mathbf{0}}^{\mathbf{k}-\mathbf{j}}\right) \rightarrow H_{*}\left(\vec{P}\left(K_{\mathbf{j}^{\prime}}\right)_{\mathbf{0}}^{\mathbf{k}-\mathbf{j}^{\prime}}\right) \\
(i n c l)_{*}: & \left.H_{*}\left(F_{\mathbf{j}} \vec{P}(K)_{\mathbf{0}}^{\mathbf{k}}\right)\right) \rightarrow H_{*}\left(F_{\mathbf{j}^{\prime}} \vec{P}(K)_{\mathbf{0}}^{\mathbf{k}}\right)
\end{aligned}
$$

define functors $A_{*}\left(K_{(-)}\right), H_{*}\left(\vec{P}\left(K_{(-)}\right)_{\mathbf{0}}^{\mathbf{k}-(-)}, F_{(-)} \vec{P}(K)_{\mathbf{0}}^{\mathbf{k}}: \mathcal{J} \rightarrow \mathbf{A} \mathbf{b}_{*} ;\right.$ compare Propositions 3.1 and 2.6.

Proposition 3.3 If $[\mathbf{k}-\mathbf{1}, \mathbf{k}] \subseteq K$, the homomorphism $\Phi_{K}$ is the composition

$$
\begin{aligned}
& A_{*}(K) \stackrel{\simeq}{\longleftarrow} \operatorname{colim}_{\mathbf{j} \in \mathcal{J}} A_{*}\left(K_{\mathbf{j}}\right) \stackrel{\operatorname{colim}_{\mathbf{j}} \Phi_{K_{\mathbf{j}}}}{\longrightarrow} \operatorname{colim}_{\mathbf{j} \in \mathcal{J}} H_{*}\left(\vec{P}\left(K_{\mathbf{j}}\right)_{\mathbf{0}}^{\mathbf{k}-\mathbf{j}}\right) \\
& \stackrel{\operatorname{colim}_{\mathbf{j}}\left(i_{\mathbf{k}-\mathbf{j}}^{\mathbf{k}}\right)_{*}}{\longrightarrow} \operatorname{colim}_{\mathbf{j} \in \mathcal{J}} H_{*}\left(F_{\mathbf{j}} \vec{P}(K)_{\mathbf{0}}^{\mathbf{k}}\right) \stackrel{Q}{\longrightarrow} H_{*}\left(\operatorname{hocolim}_{\mathbf{j} \in \mathcal{J}} F_{\mathbf{j}} \vec{P}(K)_{\mathbf{0}}^{\mathbf{k}}\right) \stackrel{\simeq}{\rightarrow} H_{*}\left(\vec{P}(K)_{\mathbf{0}}^{\mathbf{k}}\right) .
\end{aligned}
$$

with $Q$ the colimit of the maps $Q_{\mathbf{j}}: H_{*}\left(F_{\mathbf{j}} \vec{P}(K)_{\mathbf{0}}^{\mathbf{k}}\right) \rightarrow H_{*}\left(\operatorname{hocolim}_{\mathbf{j} \in \mathcal{J}} F_{\mathbf{j}} \vec{P}(K)_{\mathbf{0}}^{\mathbf{k}}\right)$, $\mathbf{j} \in \mathcal{J}$. Moreover, all these homomorphisms are isomorphisms.

Proof It is easy to check that the homomorphism colim $\mathbf{j} \in \mathcal{J} A_{*}\left(K_{\mathbf{j}}\right) \rightarrow A_{*}(K)$ induced by inclusions $\varphi_{K_{\mathbf{j}}}^{K}$ is an isomorphism - since $[\mathbf{k}-\mathbf{1}, \mathbf{k}] \subseteq K$. Thus, $A_{*}(K)$ is generated by cube sequences $\left[\mathbf{a}^{*}\right]$ in $K_{\mathbf{j}}$. Now the conclusion follows from Proposition 3.1 applied for pairs $K_{\mathbf{j}} \subseteq K$. Furthermore, $\operatorname{colim}_{\mathbf{j}} \Phi_{K_{\mathbf{j}}}$ is an isomorphism by the induction hypothesis, $\operatorname{colim}_{\mathbf{j}}\left(i_{\mathbf{k}-\mathbf{j}}^{\mathbf{k}}\right)_{*}$ by Proposition 2.6 and for the last isomorphism by (2.2). We are left to show that also $Q$ is an isomorphism; this will be achieved in Proposition 3.6.

Proposition 3.4 The compositions

$$
A_{*}\left(K_{\mathbf{j}}\right) \stackrel{\Phi_{K_{\mathbf{j}}}}{\longrightarrow} H_{*}\left(\vec{P}\left(K_{\mathbf{j}}\right)_{\mathbf{0}}^{\mathbf{k}-\mathbf{j}}\right) \stackrel{\left(i_{\mathbf{k}-\mathbf{j}}^{\mathbf{k}}\right)_{*}}{\longrightarrow} H_{*}\left(F_{\mathbf{j}} \vec{P}(K)_{\mathbf{0}}^{\mathbf{k}}\right)
$$

define a natural equivalence of functors $A_{*}\left(K_{(-)}\right)$and $H_{*}\left(F_{(-)} \vec{P}(K)_{\mathbf{0}}^{\mathbf{k}}\right)$ from $\mathcal{J}$ into the category of graded abelian groups.

Proof Both homomorphisms are isomorphisms by the inductive hypothesis and Proposition 2.6. The naturality of the transformations is a consequence of Propositions 3.1 and 2.6.

Proposition 3.5 For every $t \geqslant 0$, the functor $A_{t}\left(K_{(-)}\right)$is a projective object in the category of functors $\mathcal{J} \rightarrow \mathbf{A b}$.

Proof If $n-2$ does not divide $t$, then by definition $A_{t}\left(K_{(-)}\right)=0$ is projective. Assume that $t=(n-2) q, q \in \mathbb{Z}$. We find a presentation of $A_{t}\left(K_{(-)}\right)$as a direct sum 
of projective summands: Within the set $C S_{q}(K)$ of cube sequences in $K$ (cf Sect. 1.4) let

$$
X_{\mathbf{j}}=C S_{q}\left(K_{\mathbf{j}}\right) \backslash \bigcup_{\mathbf{j}<\mathbf{h} \in \mathcal{J}} C S_{q}\left(K_{\mathbf{h}}\right)
$$

Next, define functors $M_{\mathbf{j}}: \mathcal{J} \rightarrow \mathbf{A b}$ by

$$
M_{\mathbf{j}}(\mathbf{h})= \begin{cases}\mathbb{Z}\left[X_{\mathbf{j}}\right] & \text { if } \mathbf{h} \leqslant \mathbf{j} \\ 0 & \text { otherwise, }\end{cases}
$$

the morphisms are identities whenever possible, and trivial otherwise. Immediately from the definitions we obtain that $C S_{q}\left(K_{\mathbf{j}}\right)=\bigcup_{\mathbf{h} \geqslant \mathbf{j}} X_{\mathbf{h}}$ and hence $A_{t}\left(K_{(-)}\right) \cong$ $\bigoplus_{\mathbf{j} \in \mathcal{J}} M_{\mathbf{j}}$.

For an arbitrary functor $N: \mathcal{J} \rightarrow \mathbf{A b}$ the set of transformations $\operatorname{Hom}_{\mathcal{J}}\left(M_{\mathbf{j}}, N\right)$ is naturally isomorphic to $\operatorname{Hom}\left(\mathbb{Z}\left[X_{\mathbf{j}}\right], N(\mathbf{j})\right)$. Therefore the projectivity of $\mathbb{Z}\left[X_{\mathbf{j}}\right]$ implies that the functors $M_{\mathbf{j}}$ are also projective. As a consequence, $A_{t}\left(K_{(-)}\right) \cong$ $\bigoplus_{\mathbf{j} \in \mathcal{J}} M_{\mathbf{j}}$ is projective.

\section{Proposition 3.6 The homomorphism}

$$
Q: \operatorname{colim}_{\mathbf{j} \in \mathcal{J}} H_{*}\left(F_{\mathbf{j}} \vec{P}(K)_{\mathbf{0}}^{\mathbf{k}}\right) \longrightarrow H_{*}\left(\operatorname{hocolim}_{\mathbf{j} \in \mathcal{J}} F_{\mathbf{j}} \vec{P}(K)_{\mathbf{0}}^{\mathbf{k}}\right)
$$

is an isomorphism.

Proof Following Bousfield and Kan [3, XII.5.7], there is a spectral sequence

$$
E_{s, t}^{2}=\operatorname{colim}_{\mathcal{J}_{k}}^{s} H_{t}\left(F_{\mathbf{j}} \vec{P}(K)_{\mathbf{0}}^{\mathbf{k}}\right) \Rightarrow H_{*}\left(\operatorname{hocolim}_{\mathbf{j} \in \mathcal{J}} F_{\mathbf{j}} \vec{P}(K)_{\mathbf{0}}^{\mathbf{k}}\right)
$$

where colim $s$ stands for $s$-th left derived functor of colim. In fact, $E_{s, t}^{2}=0$ for $s>0$ since

$$
H_{t}\left(F_{(-)} \vec{P}(K)_{\mathbf{0}}^{\mathbf{k}}\right) \simeq A_{t}\left(K_{(-)}\right)
$$

is projective (Propositions 3.4 and 3.5). Hence the spectral sequence degenerates to the isomorphism $\operatorname{colim}_{\mathbf{j} \in \mathcal{J}} H_{*}\left(F_{\mathbf{j}} \vec{P}(K)_{\mathbf{0}}^{\mathbf{k}}\right) \cong H_{*}\left(\operatorname{hocolim}_{\mathbf{j} \in \mathcal{J}} F_{\mathbf{j}} \vec{P}(K)_{\mathbf{0}}^{\mathbf{k}}\right)$.

Corollary 3.7 Assume that $[\mathbf{k}-\mathbf{1}, \mathbf{k}] \subseteq K$ and $\Phi_{K_{\mathbf{j}}}$ is an isomorphism for $\mathbf{j} \in \mathcal{J}$. Then $\Phi_{K}$ is an isomorphism, as well.

Proof This follows immediately from Propositions 3.3 and 3.6.

\subsubsection{The case $[\mathbf{k}-\mathbf{1}, \mathbf{k}] \nsubseteq K$}

Denote $L:=K \cup[\mathbf{k}-\mathbf{1}, \mathbf{k}]$ and denote $\hat{\mathcal{J}}:=\hat{\mathcal{J}}_{n-1}$, and $\mathcal{J}:=\mathcal{J}_{n-1}$ with objects

$$
\mathrm{Ob}(\hat{\mathcal{J}})=\left\{\mathbf{j} \in\{0,1\}^{n}: \mathbf{0}<\mathbf{j}<\mathbf{1}\right\} \subseteq\left\{\mathbf{j} \in\{0,1\}^{n}: \mathbf{0}<\mathbf{j}\right\}=\mathrm{Ob}(\mathcal{J})
$$


Their nerves $N \hat{\mathcal{J}}, N \mathcal{J}$ have geometric realizations $S^{n-2} \cong \partial \Delta^{n-1} \cong|N \hat{\mathcal{J}}| \subset$ $|N \mathcal{J}| \cong \Delta^{n-1}$. Consider the sequence of cofibrations

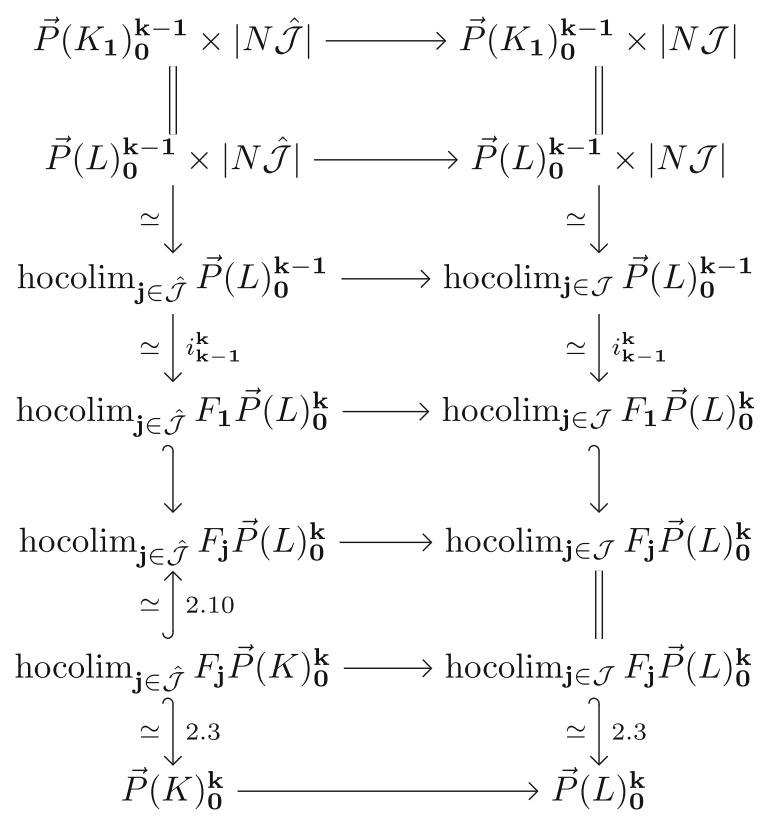

In all the squares of the diagram apart from the middle one the vertical maps are homotopy equivalences; hence the cofibres are also homotopy equivalent. One can easily check, using the construction of the homotopy colimit, that the maps in the middle square induce a homeomorphism between cofibres of type $F_{\mathbf{1}} \vec{P}(L)_{\mathbf{0}}^{\mathbf{k}} \times S^{n-1}$. The diagram above induces a transfomation between associated homology long exact sequences. In particular, the following diagram

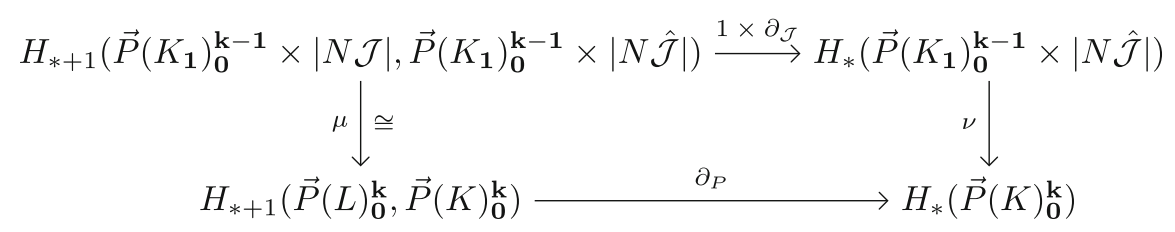

is commutative, where

$$
\partial_{\mathcal{J}}: H_{*+1}(|N \mathcal{J}|,|N \hat{\mathcal{J}}|) \rightarrow H_{*}(|N \hat{\mathcal{J}}|)
$$

is the differential of the long homology exact sequence of the pair $(|N \mathcal{J}|,|N \hat{\mathcal{J}}|)$ and where $\mu$ is an isomorphism. Let $\Psi$ be the composition 


$$
\begin{aligned}
& A_{*-(n-2)}\left(K_{\mathbf{1}}\right) \stackrel{\Phi_{K_{\mathbf{1}}}}{\longrightarrow} H_{*-(n-2)} \vec{P}\left(K_{\mathbf{1}}\right)_{\mathbf{0}}^{\mathbf{k}-\mathbf{1}} \\
& \quad \stackrel{\times \partial_{\mathcal{J}}^{-1}\left(x_{\mathbf{k}}\right)}{\longrightarrow} H_{*+1}\left(\vec{P}\left(K_{\mathbf{1}}\right)_{\mathbf{0}}^{\mathbf{k}-\mathbf{1}} \times\left|N \mathcal{J}^{\prime}\right|, \vec{P}\left(K_{\mathbf{1}}\right)_{\mathbf{0}}^{\mathbf{k}-\mathbf{1}} \times|N \mathcal{J}|\right) \stackrel{\mu}{\rightarrow} H_{*+1}\left(\vec{P}(L)_{\mathbf{0}}^{\mathbf{k}}, \vec{P}(K)_{\mathbf{0}}^{\mathbf{k}}\right),
\end{aligned}
$$

where $x_{\mathbf{k}} \in H_{n-2}(|N \hat{\mathcal{J}}|)$ is a fixed generator. By the inductive assumption, $\Phi_{K_{1}}$ is an isomorphism and so is $\Psi$. Define

$$
\varrho: A_{*-(n-2)}\left(K_{1}\right) \ni\left[\mathbf{a}^{1} \ll \cdots \ll \mathbf{a}^{r}\right] \mapsto\left[\mathbf{a}^{1} \ll \cdots \ll \mathbf{a}^{r} \ll \mathbf{k}\right] \in A_{*}(K),
$$

Proposition 3.8 The diagram

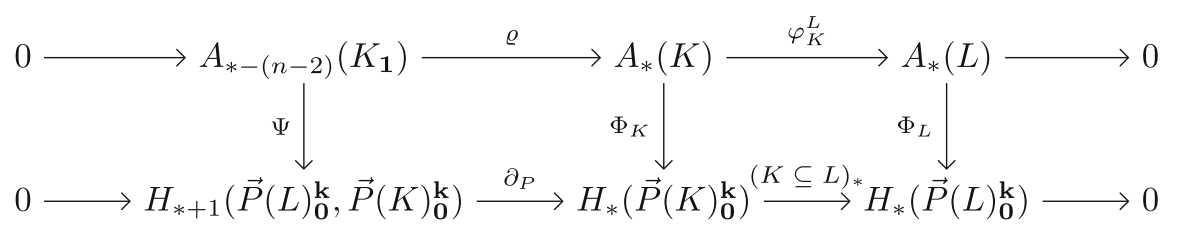

is commutative, and it has exact rows. Moreover, all vertical homomorphisms are isomorphisms.

Proof Exactness of the upper row follows immediately from definitions. The right square commutes by Proposition 3.1. Since $\Phi_{L}$ is an isomorphism (by Proposition 3.3), the composition $\Phi_{L} \circ \varphi_{K}^{L}$ is surjective. Hence $(K \subseteq L)_{*}$ is also surjective and this implies exactness of the lower row. For every cube sequence [ $\left.\mathbf{a}^{*}\right]$ in $K_{\mathbf{1}}$, we have

$$
\begin{aligned}
& \partial_{P}\left(\Psi\left(\left[\mathbf{a}^{*}\right]\right)\right)=\partial_{P}\left(\mu\left(\Phi_{K_{\mathbf{1}}}\left(\left[\mathbf{a}^{*}\right]\right) \times \partial_{\mathcal{J}}^{-1}\left(x_{\mathbf{k}}\right)\right)\right)=v\left(\Phi_{K_{\mathbf{1}}}\left(\left[\mathbf{a}^{*}\right]\right) \times x_{\mathbf{k}}\right) \\
& \quad=v\left(\Phi_{K_{\mathbf{1}}}\left(\left[\mathbf{a}^{*}\right]\right) \times \Phi_{[\mathbf{k}-\mathbf{1}, \mathbf{k}]_{(n-1)}}([\mathbf{k}])\right)=\Phi_{K}\left(\left[\mathbf{a}^{*} \ll \mathbf{k}\right]\right)=\Phi_{K}\left(\varrho\left(\left[\mathbf{a}^{*}\right]\right)\right) .
\end{aligned}
$$

Hence the left square commutes. Finally, since both $\Psi$ and $\Phi_{L}$ are isomorphisms, $\Phi_{K}$ is an isomorphism by the Five Lemma.

Proof of Theorem 3.2 Induction starts for $\mathbf{k} \gg \mathbf{0}$ as stated immediately after the statement of Theorem 3.2. Assume that the theorem holds for all $\mathbf{l}<\mathbf{k}$. By Corollary 3.7, $\Phi_{K}$ is an isomorphism whenever $[\mathbf{k}-\mathbf{1}, \mathbf{k}] \subseteq K$ and by Proposition 3.8, it holds for any $K \subseteq[\mathbf{0}, \mathbf{k}]$.

\subsection{A generalization}

The main Theorem 3.2 applies of course also to spaces that are homotopy equivalent to the path spaces $\vec{P}(K)_{\mathbf{0}}^{\mathbf{k}}$. To obtain such spaces consider a functor $Z: \mathbb{Z}_{+}^{n} \rightarrow$ Top from the poset of non-negative $n$-dimensional vectors (regarded as a category) obeying to

$$
Z(\mathbf{k}) \begin{cases}\text { contractible, } & \prod_{i} k_{i}=0 \\
\simeq \operatorname{hocolim}_{j \in \mathcal{C}_{\mathbf{k}}} Z(\mathbf{k}-\mathbf{j}), \mathcal{C}_{\mathbf{k}}=\left\{\begin{array}{ll}
\mathcal{J},[\mathbf{k}-\mathbf{1}, \mathbf{k}] \subset K \\
\hat{\mathcal{J}},[\mathbf{k}-\mathbf{1}, \mathbf{k}] \not \subset K
\end{array}, \prod_{i} k_{i} \neq 0\right.\end{cases}
$$


A particular simple such functor functor $Z_{0}$ can be constructed recursively by

$$
Z_{0}(\mathbf{k})= \begin{cases}*(\text { a one point space }) & \prod_{i} k_{i}=0 \\ \operatorname{hocolim}_{j \in \mathcal{C}_{\mathbf{k}}} Z(\mathbf{k}-\mathbf{j}) & \prod_{i} k_{i} \neq 0\end{cases}
$$

with $\mathcal{C}_{\mathbf{k}}$ as above.

Proposition 3.9 Functors $Z_{i}, Z_{j}: \mathbb{Z}_{+}^{n} \rightarrow$ Top obeying to (3.6) yield homotopy equivalent spaces $Z_{i}(\mathbf{k}) \simeq Z_{j}(\mathbf{k})$ for all $\mathbf{k} \in \mathbb{Z}_{+}^{n}$.

Proof This can be seen inductively (for $Z_{j}=Z_{0}$ ) starting from constant maps $Z_{j}(\mathbf{k}) \rightarrow Z_{0}(\mathbf{k})$ for $\prod_{i} k_{i}=0$ extending to

$$
Z_{i}(\mathbf{k}) \simeq \operatorname{hocolim}_{j \in \mathcal{C}_{\mathbf{k}}} Z_{i}(\mathbf{k}-\mathbf{j}) \simeq \operatorname{hocolim}_{j \in \mathcal{C}_{\mathbf{k}}} Z_{0}(\mathbf{k}-\mathbf{j}) \simeq Z_{o}(\mathbf{k})
$$

In particular, the functor $Z(\mathbf{k})=\vec{P}(K)_{\mathbf{0}}^{\mathbf{k}}$ obeys to (3.6), and hence $\vec{P}(K)_{\mathbf{0}}^{\mathbf{k}} \simeq Z_{0}(\mathbf{k})$ for all $\mathbf{k} \in \mathbb{Z}_{+}^{n}$. We shall now present a "sub"functor $Z_{1}$ also obeying to (3.6) that can serve to motivate the main Theorem 3.2:

Cube sequences between $\mathbf{0}$ and $\mathbf{k}$ are partially ordered by inclusion. A cube sequence is maximal if $\mathbf{a}^{i} \ll \mathbf{a}^{i+1}-\mathbf{1}$ for all $i$. Let $\bar{C} S(K)_{\mathbf{0}}^{\mathbf{k}}$ denote the set of maximal cube sequences between $\mathbf{0}$ and $\mathbf{k}$. For a maximal cube sequence $\mathbf{a}^{*}$, let $P\left(\mathbf{a}^{*}\right)=\prod_{j=1}^{r} \vec{P}(K)_{\mathbf{a}^{j-1}}^{\mathbf{a}^{j}} \times \vec{P}(K)_{\mathbf{a}^{j}}^{\mathbf{a}^{j+1}-\mathbf{1}}$; by maximaity, the latter factor is always contractible, and hence $P\left(\mathbf{a}^{*}\right) \simeq \prod_{j=1}^{r} \vec{P}(K)_{\mathbf{a}^{j-1}}^{\mathbf{a}^{j}} \simeq \prod_{j=1}^{r}\left(S^{n-2}\right)^{r}$.

Concatenation defines maps $\bar{c}\left(\mathbf{a}^{*}\right): P\left(\mathbf{a}^{*}\right) \rightarrow \vec{P}(K)_{\mathbf{0}}^{\mathbf{k}}$ assembling to $\bar{c}:$

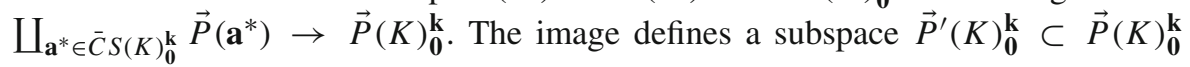
of d-paths through integral points that - alternatingly - have at least one coordinate in common or for which every coordinate is the successor of the previous one.

Corollary 3.10 Inclusion $\vec{P}^{\prime}(K)_{\mathbf{0}}^{\mathbf{k}} \subset \vec{P}(K)_{\mathbf{0}}^{\mathbf{k}}$ is a homotopy equivalence for all $\mathbf{0} \leq \mathbf{k}$.

Proof It is easy to check that the functor $Z_{1}(\mathbf{k})=\vec{P}^{\prime}(K)_{\mathbf{0}}^{\mathbf{k}}$ obeys to (3.6). Apply Proposition 3.9.

\section{The cohomology ring of the path space $\vec{P}(K)_{0}^{\mathrm{k}}$}

Fix $\mathbf{k} \in \mathbb{Z}^{n}$ and a Euclidean cubical complex $K \subseteq[\mathbf{0}, \mathbf{k}]$ containing its $(n-1)$ skeleton. As proven in the previous section 3, the homology of the path space $\vec{P}(K)_{\mathbf{k}}^{\mathbf{0}}$ is isomorphic, as a graded group, to $A_{*}(K)$. Since this group is free, the cohomology of $\vec{P}(K)_{\mathbf{k}}^{\mathbf{0}}$ is isomorphic to its dual, i.e. there is a sequence of isomorphisms

$$
\Phi^{K}: H^{*}\left(\vec{P}(K)_{\mathbf{k}}^{\mathbf{0}}\right) \cong \operatorname{Hom}\left(H_{*}\left(\vec{P}(K)_{\mathbf{k}}^{\mathbf{0}}\right), \mathbb{Z}\right) \stackrel{\operatorname{Hom}\left(\Phi_{K}, \mathbb{Z}\right)}{\longrightarrow} A^{*}(K):=\operatorname{Hom}\left(A_{*}(K), \mathbb{Z}\right) .
$$

Let $Z^{*}(K)$ denote the free graded exterior $\mathbb{Z}$-algebra with generators the cube sequences $[\mathbf{0} \ll \mathbf{l} \leq \mathbf{k}],[\mathbf{l}-\mathbf{1}, \mathbf{l}] \not \subset K$ (of length 1 ). Let $I(K)$ denote the ideal generated by products $\mathbf{l}_{1} \mathbf{l}_{2}$ with $\mathbf{l}_{1} \ll \mathbf{l}_{2}$ and $\mathbf{l}_{2} \ll \mathbf{l}_{1}$. Let $F^{*}(K)$ denote the quotient algebra $F^{*}(K)=Z^{*}(K) / I^{*}(K)$, a free abelian group with the cube sequences [a*] 
in $K$ as basis. Moreover, we can provide $A^{*}(K)$ with a ring structure via the $\mathbb{Z}$-module isomorphism $\Psi^{K}: A^{*}(K) \rightarrow F^{*}(K), \phi \mapsto \sum_{\mathbf{a}^{*} \in C S(K)} \phi\left(\mathbf{a}^{*}\right) \mathbf{a}^{*}$.

Proposition 4.1 The map $\Psi^{K} \circ \Phi^{K}: H^{*}\left(\vec{P}(K)_{\mathbf{k}}^{\mathbf{0}}\right) \rightarrow F^{*}(K)$ is a graded ring isomorphism.

Proof Fix a cube sequence $\mathbf{a}^{*}$ in $K$, giving rise to inclusion [by concatenation, as in (3.1)] $c\left(\mathbf{a}^{*}\right): \prod_{i=1}^{r} \vec{P}(K)_{\mathbf{a}^{i}-\mathbf{1}}^{\mathbf{a}^{i}} \rightarrow \vec{P}(K)_{\mathbf{0}}^{\mathbf{k}}$. To $\mathbf{a}^{*}$ corresponds

- a graded abelian group $i\left(\mathbf{a}^{*}\right): A_{*}\left(\mathbf{a}^{*}\right) \subset A_{*}(K)$ generated by the set of $s u b$-cube sequences of $\mathbf{a}^{*}$ with $i\left(\mathbf{a}^{*}\right)$ the inclusion homomorphism

- a free graded exterior algebra $F^{*}\left(\mathbf{a}^{*}\right)=Z^{*}\left(\mathbf{a}^{*}\right)$ with generators $\mathbf{a}^{i}$ and a projection homomorphism $p\left(\mathbf{a}^{*}\right): F^{*}(K) \rightarrow F^{*}\left(\mathbf{a}^{*}\right)$ and

- an additive isomorphism $\Psi\left(\mathbf{a}^{*}\right): A^{*}\left(\mathbf{a}^{*}\right) \rightarrow F^{*}\left(\mathbf{a}^{*}\right)$.

Moreover, the group isomorphism $\Phi\left(\mathbf{a}^{*}\right): A_{*}\left(\mathbf{a}^{*}\right) \rightarrow H_{*}\left(\prod_{i=1}^{r} \vec{P}(K)_{\mathbf{a}^{i}-1}^{\mathbf{a}^{i}}\right)$ has a dual $\Phi\left(\mathbf{a}^{*}\right)^{*}: H^{*}\left(\prod_{i=1}^{r} \vec{P}(K)_{\mathbf{a}^{i}-1}^{\mathbf{a}^{i}}\right) \rightarrow A^{*}\left(\mathbf{a}^{*}\right)$ fitting into the commutative diagram

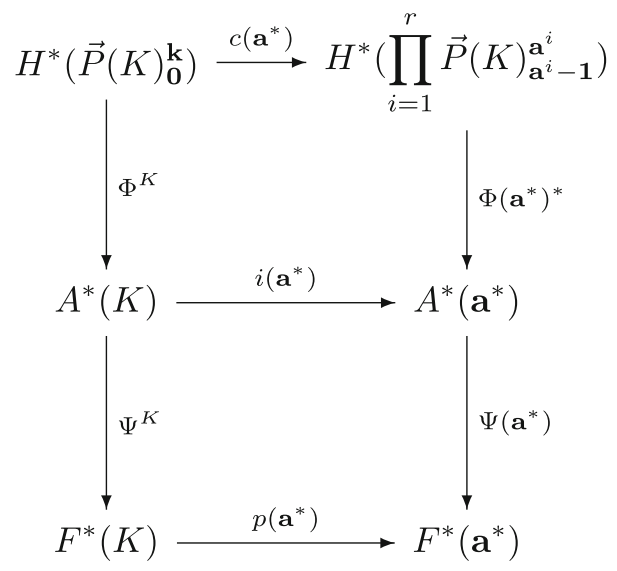

All vertical maps are isomorphisms of abelian groups, and all maps, apart from possibly $\Phi^{K}$, are ring homomorphisms.

The assembly map $\bigoplus_{\mathbf{a}^{*} \in C S(K)} A_{*}(\mathbf{a}) \rightarrow A_{*}(K)$ is clearly surjective whence its dual $\bigoplus_{\mathbf{a}^{*} \in C S(K)} i\left(\mathbf{a}^{*}\right): \bigoplus_{\mathbf{a}^{*} \in C S(K)} A^{*}(K) \rightarrow \bigoplus_{\mathbf{a}^{*} \in C S(K)} A^{*}\left(\mathbf{a}^{*}\right)$ injects. Hence, $\Phi^{K}$ is a ring isomorphism, as well.

\section{References}

1. Brown, R., Higgins, P.J.: On the algebra of cubes. J. Pure Appl. Algebra 21, 233-260 (1981)

2. Brown, R., Higgins, P.J., Sivera, R.: Nonabelian algebraic topology. Filtered spaces, crossed complexes, cubical homotopy groupoids. In: EMS Tracts in Mathematics, vol. 15. European Mathematical Society (EMS), Zürich (2011)

3. Bousfield, A.K., Kan, D.M.: Homotopy limits, completions and localizations. Lecture Notes in Mathematics, vol. 304. Springer, New York (1972) 
4. Dijkstra, E.W.: Co-operating sequential processes. In: Genuys, F. (ed.) Programming Languages, pp. 43-110. Academic Press, New York (1968)

5. Dijkstra, E.W.: Hierarchical ordering of sequential processes. Acta Inform. 1, 115-138 (1971)

6. Fajstrup, L.: Trace Spaces of Directed Tori with Rectangular Holes. Math. Struct. Comput. Sci. FirstView, 1-23. doi:10.1017/S096012951300169

7. Fajstrup, L., Goubault, É., Raussen, M.: Algebraic topology and concurrency. Theor. Comput. Sci. 357, 241-278 (2006) (revised version of Aalborg University, preprint, 1999)

8. Grandis, M.: Directed homotopy theory, I. The fundamental category. Cahiers Top. Geom. Diff. Categ. 44, 281-316 (2003)

9. Grandis, M.: Directed Algebraic Topology. Cambridge University Press, Cambridge (2009)

10. Pratt, V.: Modelling concurrency with geometry. In: Proceedings of the 18th ACM Symposium on Principles of Programming Languages, pp. 311-322 (1991)

11. Raussen, M.: On the classification of dipaths in geometric models for concurrency. Math. Struct. Comput. Sci. 10, 427-457 (2000)

12. Raussen, M.: Trace spaces in a pre-cubical complex. Topol. Appl. 156, 1718-1728 (2009)

13. Raussen, M.: Simplicial models for trace spaces. Algebra Geom. Topol. 10, 1683-1714 (2010)

14. Raussen, M.: Simplicial models for trace spaces II: general higher dimensional automata. Algebra Geom. Topol. 12, 1545-1565 (2012)

15. Raussen, M.: Execution spaces for simple higher dimensional automata. Appl. Algebra Eng. Commun. Comput. 23, 59-84 (2012)

16. Segal, G.: Classifying spaces and spectral sequences. Inst. Hautes Etudes Sci. Publ. Math. 34, 105-112 (1968)

17. van Glabbeek, R.J.: On the expressiveness of higher dimensional automata. Theor. Comput. Sci. 368, 168-194 (2006)

18. Ziemiański, K.: A cubical model for path spaces in d-simplicial complexes. Topol. Appl. 159, 2127$2145(2012)$ 